
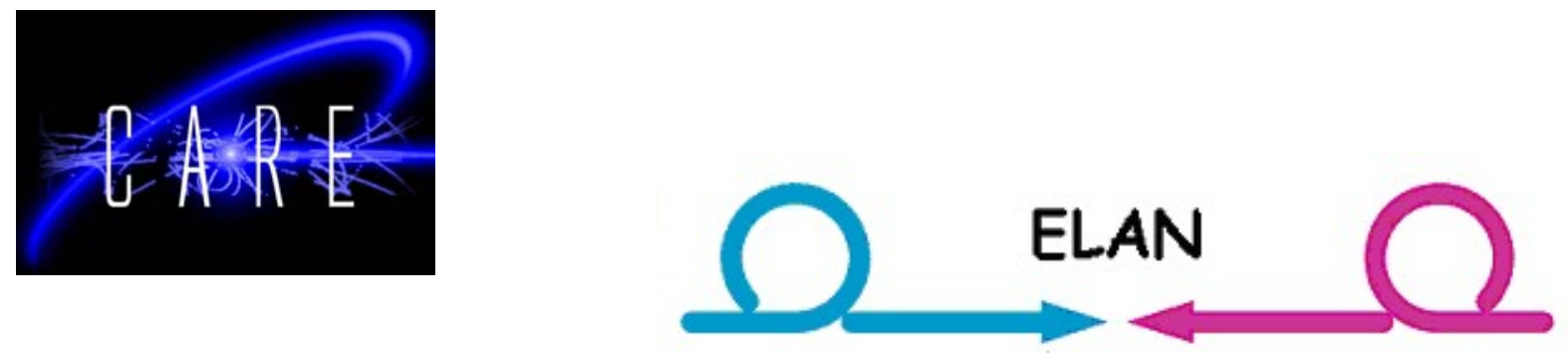

\title{
CONSIDERATIONS ON THE DESIGN OF THE DECELERATOR OF THE CLIC TEST FACILITY (CTF3)
}

D. Schulte, I. Syratchev

CERN, Geneva, Switzerland

\begin{abstract}
One of the main aims of the CLIC Test Facility (CTF3) is to study the beam stability in the drive beam decelerator and to benchmark the performance against beam simulation codes. Particular challenges come from the large drive beam energy spread, the strong wakefields and potential beam losses. The development towards a decelerator design and the required instrumentation is described in this paper.
\end{abstract}

Contribution to the PAC05, Knoxville, USA

Work supported by the European Community-Research Infrastructure Activity under the FP6 " Structuring the European Research Area » programme (CARE, contract number RII3-CT2003-506395). 
EUROPEAN ORGANIZATION FOR NUCLEAR RESEARCH

CERN - AB Department

CERN-AB-2005-050

CLIC Note 634

\title{
CONSIDERATIONS ON THE DESIGN OF THE DECELERATOR OF THE CLIC TEST FACILITY (CTF3) \\ D. Schulte, I. Syratchev \\ CERN, Geneva, Switzerland
}

\begin{abstract}
One of the main aims of the CLIC Test Facility (CTF3) is to study the beam stability in the drive beam decelerator and to benchmark the performance against beam simulation codes. Particular challenges come from the large drive beam energy spread, the strong wakefields and potential beam losses. The development towards a decelerator design and the required instrumentation is described in this paper.
\end{abstract}

Presented at

PAC 2005, Knoxville, USA,

May 16-20, 2005

Geneva, Switzerland

June 2005 


\title{
EU contract number RII3CT-2003-506395 \\ Considerations on the Design of the Decelerator of the CLIC Test Facility \\ (CTF3)
}

\author{
D. Schulte and I. Syratchev, CERN, Switzerland
}

\begin{abstract}
One of the main aims of the CLIC Test Facility (CTF3) is to study the beam stability in the drive beam decelerator and to benchmark the performance against beam simulation codes. Particular challenges come from the large drive beam energy spread, the strong wakefields and potential beam losses. The development towards a decelerator design and the required instrumentation is described in this paper.
\end{abstract}

\section{INTRODUCTION}

In the CLIC concept a high-current low-energy drive beam is decelerated in power extraction and transfer structures (PETS) in order to generate the accelerating RF power for the main beam. A CLIC test facility (CTF3) is under construction at CERN in order to demonstrate the feasibility of the generation of the drive beam. This facility should also demonstrate that it is possible to extract the drive beam power in a decelerator without the beam becoming unstable. To this end a test beam line (TBL) will be built in which the CTF3 drive beam can be decelerated. The layout of this beam line will follow the same layout as the one for CLIC.

\section{DESIGN STRATEGY}

In CLIC, the drive beam decelerator consists of a series of FODO cells with a constant length. The layout of each cell is shown in Fig. 1. The focusing is adjusted in a way that the particles that are decelerated the most always experience the same phase advance per cell; therefore, the higher energy particles are less focused. However, due to their smaller geometric emittance, their envelope remains within that of the lowest energy particles. The same layout will be used for the TBL.

In table 1, the main parameters of the drive beam which could possibly be used in the TBL are listed. The beam current is significantly lower than in CLIC. The initial beam energy in the TBL is well below the final energy after

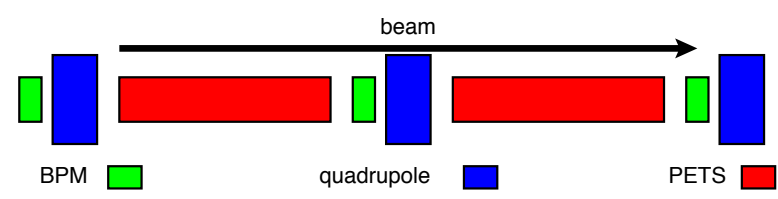

Figure 1: Layout of the basic decelerator FODO cell.
Table 1: The main parameters of the CTF3 drive beam before the TBL. For comparison potential parameters for the CLIC drive beam before the decelerator are also given.

\begin{tabular}{|c|c|c|c|}
\hline & Meaning & Value TBL & Value CLIC \\
\hline$E_{0}$ & initial beam energy & $150 \mathrm{MeV}$ & $2070 \mathrm{MeV}$ \\
$N$ & bunch charge & $1.5 \times 10^{10} e$ & $7.5 \times 10^{10} e$ \\
$\Delta z$ & bunch distance & $20 \mathrm{~mm}$ & $20 \mathrm{~mm}$ \\
$\sigma_{z}$ & RMS bunch length & $400 \mu \mathrm{m}$ & $400 \mu \mathrm{m}$ \\
$\epsilon_{x}$ & norm. $x$-emittance & $150 \mu \mathrm{m}$ & $150 \mu \mathrm{m}$ \\
$\epsilon_{y}$ & norm. $y$-emittance & $150 \mu \mathrm{m}$ & $150 \mu \mathrm{m}$ \\
$n_{b}$ & bunches per pulse & $\approx 2000$ & $\approx 1000$ \\
\hline
\end{tabular}

the drive beam decelerator in CLIC. On the one hand this makes the experiment more complicated, since in the TBL the assumption that the beam size is much smaller than the PETS aperture is not valid. On the other hand, the low beam current leads to less coupling to the impedances. Another main difference is the pulse length, which is twice longer in CTF3 compared to the new CLIC parameters. This helps to identify modes which have little damping.

Two approaches to the demonstration can be used. Firstly, one can determine the wakefield level at which the drive beam in CLIC would be unstable and then design the TBL in such a way that this level of wakefield would be visible. The most reliable results would be achieved using the same PETS design in both cases. Secondly, one can design the TBL to be able to measure wakefields which would even be acceptable in CLIC. This would explicitly benchmark the modelling, while in the former method one could show the stability without determining more than an upper limit for the wakefields. While the second method is more desirable it requires a relatively high wakefield level; the former method may thus be simpler.

\section{TBL MODELLING}

For the simulation of the individual structures HFFS [1] and GdfidL [2] are used. Their results are transformed into a simple model of the transverse and longitudinal wakefield. These are used in the beam transport code PLACET [3].

In the case of CLIC, the PETS are overmoded with an aperture of $22.5 \mathrm{~mm}$ and an RF frequency of $30 \mathrm{GHz}$. This leads to a high group velocity of the RF modes (around $\beta=0.8$ ), which can lead to important effects in the short $(0.6 \mathrm{~m})$ structures. The longitudinal wakefield is modelled as a single RF mode. In the beam dynamics simulations, 


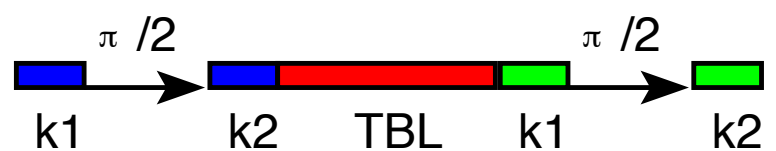

Figure 2: Schematic layout of the measurement set-up. Two kickers which are spaced by a betatron phase advance of $\pi / 2$ can kick the beam at any phase before the TBL. Two BPMs at the end of the TBL, also separated by $\pi / 2$ can detect the offsets at any phase.

the effect of the high group velocity of this mode is modelled, but the small effect of the damping of this mode is neglected.

The transverse wakefield is also modelled using a small number of RF modes. In this case the damping is included since it has a significant effect on the beam. The amplitude of the wake generated by a driving particle of charge $q$ on a particle travelling at a distance $z$ behind is given by

$$
w_{\perp}=w_{0} q \sin (k z) \exp (-k z / 2 Q(1-\beta))
$$

Here, the mode has the amplitude $w_{0}$, the wavenumber $k$, the damping factor $Q$ and the group velocity $\beta$. The effect of the drain-out is taken into account separately by calculating the length over which the wakefield of a leading particle is seen by the following one.

For the present studies only the linear wakefields (following the above model) and linear magnetic field components are taken into account. The longitudinal motion of the particles is modelled in a simplified way. It can be quite significant at these low energies, with a up to about $1 \mathrm{~mm}$.

The beam losses have been simulated by simply removing all particles that exceed the aperture of the PETS or quadrupole at the entrance or exit of the element.

\section{DETECTION OF WAKEFIELD MODES}

In the first method, the aim will be to measure the transverse wakefield with the beam by simply offsetting the beam at the entrance of the TBL and monitoring the development of this offset along the decelerator. If no transverse wakefields were present, the amplitude of the beam offsets would only increase because of the adiabatic undamping due to the energy loss. In the presence of transverse wakefields, the amplitude can grow significantly. For easily measurable offsets, this modification of the trajectory may however lead to beam losses. These can prohibit the operation in this regime and also heavily affect the BPM measurements.

In the second method, one can try to measure the wakefield using resonant kickers and BPMs. A potential layout of the experiment is shown in Fig. 2. As mentioned above, the wakefield is expected to consist of a number of modes, which can be defined by their loss factor, $Q$-value and wavelength. A potential way to measure these modes
Table 2: The five wakefield modes of the unacceptable PETS.

\begin{tabular}{|c|c|c|c|c|}
\hline Mode & $f[\mathrm{GHz}]$ & $w_{0}\left[\mathrm{~V} / \mathrm{pCm}^{2}\right]$ & $Q$ & $\beta$ \\
\hline 1 & 28.216 & 2100 & 45.3 & 0.892 \\
2 & 35.179 & 900 & 44.7 & 0.643 \\
3 & 41.680 & 48 & 578 & 0.05 \\
4 & 46.278 & 163 & 1940 & 0.12 \\
5 & 4.898 & 20 & 4600 & 0 \\
\hline
\end{tabular}

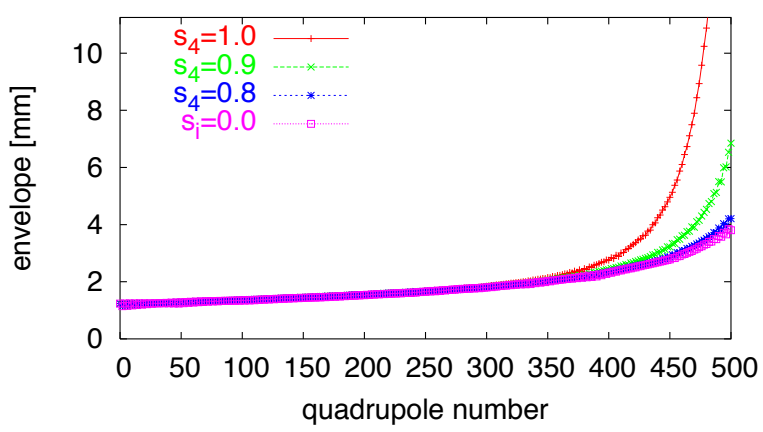

Figure 3: The envelope of a $3 \sigma$ beam with an initial offset of $\Delta y=0.3 \sigma_{y}$ along the decelerator. An unacceptable structure design has been used for the calculation to show strong effects. If the amplitude of the most dangerous mode is reduced to $80 \%$ the beam will be acceptable.

is to induce a bunch-to-bunch offset in the initial beam that has the same wavelength as the mode using the kickers 1 and 2. This bunch-to-bunch offset amplifies along the decelerator due to the beam deceleration and also due to the wakefield. At the end of the decelerator, the amplitude of the bunch-to-bunch offsets can be measured with the BPMs 1 and 2 and compared to the value expected for the case where no wakefields are present. It will be necessary to tune the frequency of the kickers and the BPMs to be able to detect all modes. Since the bunches arrive with a frequency of $15 \mathrm{GHz}$, it is sufficient to cover a $7.5 \mathrm{GHz}$ frequency band width. It is assumed that a difference of a factor of two can be detected.

\section{SIMULATION RESULTS}

\section{Using CLIC PETS}

In the simplest approach the CLIC PETS can be used in the TBL. In this case, one aims to measure any wakefield which would make the CLIC drive beam unstable. It is therefore necessary to identify at which wakefield level this occurs. For this purpose, a structure design that has been rejected due to the wakefield level was used in the simulations. The five most important transverse modes of this structure are listed in table 2.

The simulations were performed using the new drive beam decelerator layout and pulse length, see table 1 . The results show that the beam is unstable, see Fig. 3. Reducing the amplitude of the fourth mode, the most dangerous one 
EU contract number RII3CT-2003-506395

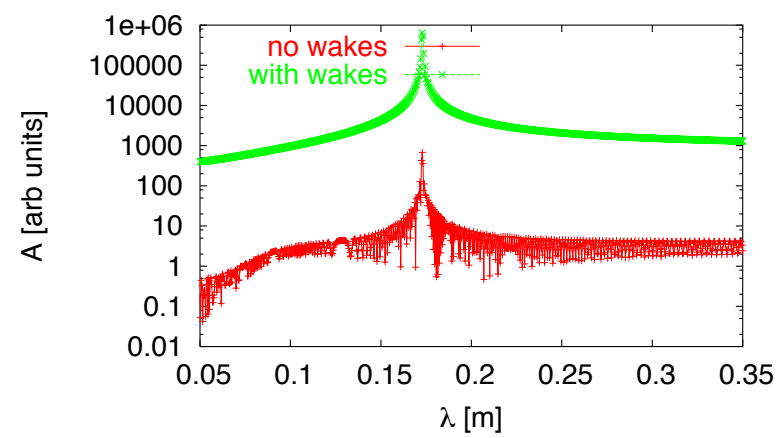

Figure 4: The final amplitude of the bunch-to-bunch offset at the wavelength of the most dangerous transverse mode for the cases where the transverse mode is present and absent.

by $20 \%$ yields a beam that is almost stable. Consequently a wakefield at this level should be visible in the TBL.

In the beginning, a TBL consisting of 28 real CLIC PETS was simulated; the total deceleration was about $12 \%$. An initial perturbation at the frequency of the fourth mode was only amplified by a factor of 2.5 as compared to the case with no wakefields. This is barely visible. Decreasing the focusing by a factor of two increased the signal to a factor of five, this is still not very large. If one uses PETS with twice the length of the nominal ones, the wakefield becomes very visible. For the nominal phase advance, the ratio of offset with and without wakefield increases to about 1000. At this stage one is already concerned about the beam stability in the TBL. In this case, the total deceleration is about $50 \%$.

In order to evaluate the visibility of other dangerous modes, the following procedure is adopted. Except for the first mode, all other mode amplitudes are set to zero. The amplitude of the first mode is scaled until a significant difference of the beam envelope becomes apparent in simulation of the CLIC drive beam decelerator. This is the case if the mode amplitude is multiplied by a factor 5 . The simulation of the TBL is repeated using the 28 long structures and the same wakefield model. In this case the wakefield increases the amplitude of the final pick-up signal by about a factor of 4 .

\section{Using Small Aperture PETS}

Another approach would be to use structures with a smaller aperture than those used for CLIC to produce a significant deceleration of the beam and increase the transverse wakefield effects. A structure has been designed for this with an aperture of $18 \mathrm{~mm}$, an impedance of $780 \Omega$ (linac), a transverse wakefield amplitude $6 \mathrm{kV} / \mathrm{mm} / \mathrm{pC}$ and group velocities of $0.78 \mathrm{c}$ for the longitudinal and $0.8 \mathrm{c}$ for the transverse wakefield. A systematic study of a number of different scaled TBL linac layouts has been made using different structure lengths, numbers and betatron phase advances per cell to determine the most meaningful exper-
CARE Conf-05-035-ELAN

iments with measureable signals. The baseline TBL consists of 18 structures each of which decelerates the beam by $7.5 \mathrm{MV}$ and the phase advance per cell is the same as that in CLIC. Scaled versions of this linac have been designed by reducing the structure length and increasing the number of structures to achieve the same overall deceleration. For each of these decelerators a set of different phase advances were used. The linacs where then cut at the longitudinal position in which a $3 \sigma$ beam would touch the aperture. Finally for all these linacs the beam was excited with the kickers and tracked. The ratio of the amplitudes of the signal in the BPMs with and without wakefields was determined. Clearly smaller phase advance and more but shorter structures enhance the difference of the BPM signal for the case with wakefields as compared to the case without. In this way it is possible to construct a decelerator with an amplification factor of more than two.

It is certainly possible to reduce the damping of the transverse modes by removing the damping material of some of the damping slots in any of the PETS designs discussed above. This would render the beam less stable. Further investigations of the structure wakefields and the beam dynamics will have to be made to assess the leverage that one can obtain by this method.

\section{CONCLUSION}

The aim of the TBL is to demonstrate the stability of the CLIC drive beam in the decelerator. To do this one needs to estimate the wakefield level at which the beam becomes unstable. One has then to ensure that this wakefield level can be detected in the TBL. A potential procedure to measure the transverse wakefields is to introduce bunchto-bunch offsets into the beam with a wavelength that can couple to the transverse mode. At the end of the TBL the bunch-to-bunch offsets can be resonantly measured at the same wavelength and the results can be compared to the value expected with no wakefields. It has been shown in this paper that a wakefield level that can affect the drive beam stability in CLIC should lead to an amplification of the bunch-to-bunch offsets by a factor of 4 or even more. Therefore one can expect to detect them in the TBL.

\section{ACKNOWLEDGEMENT}

We acknowledge the support of the European Community-Research Infrastructure Activity under the FP6 "Structuring the European Research Area" programme (CARE, contract number RII3-CT-2003-506395)

\section{REFERENCES}

[1] http://WwW.ansoft.com/products/hf/hfss/

[2] http://www.gdfidl.de/

[3] D. Schulte, CERN/PS 2000-028 (AE) (2000) 